\title{
Analysis on Drivers' Vision-psychology under the Influence of Color Difference after Pavement Pothole Repair
}

\author{
Hongjun JING*, Qian LIU, Meng GAO, Jiahao ZHANG, Xiao MU
}

\begin{abstract}
Pavement pothole repair, as one of the commonest methods used in routine road maintenance works, is effective to extend the pavement service life and increase its transport capacity. However, there are significant color differences between the old and new pavements after pothole repair, and there are few studies about the impact of color differences on driving safety. Thus, using multiple typical pavements with the pothole repair, we aim to reveal the impact of the color differences between the old and new materials on the driving safety, and to improve the safety and comfort of drivers. Tobii eye tracker and the ErgoLAB physiological recorder were used to test the driver's visual parameters when the vehicles run under the pothole repair environment, including drivers' gaze frequency, gaze duration, saccade range and heart rate variability. Results show that the color differences of the road after pothole repair have a significant influence on the drivers' vision and psychology, resulting in reduced driving safety and stability. Compared with the unrepaired road section, the gaze frequency and duration of drivers on pothole sections are significantly increased. The speed of saccade is also increased, and the saccade range is reduced. Meanwhile, the average heart rate of drivers on the pothole sections is increased by $10-20 \%$, indicating that drivers' attention and heart rate fluctuate greatly when passing through potholes, which can easily cause emotional tension and misjudgment, and thereby reduce the safety of driving. The obtained conclusions provide a significant reference for subsequent research.
\end{abstract}

Keywords: color difference after pothole repair; driving safety; eye movement characteristics; heart rate growth rate; pavement engineering

\section{INTRODUCTION}

With the rapid development of China's highway construction, the asphalt pavement has been extensively applied in road engineering, but it is influenced by the traffic overload, local pavement quality, and external natural environment. The pavement damage is unavoidable with outstanding problems. China has entered a peak phase of highway maintenance, and the highway maintenance problems will be increasingly severe. In present studies regarding the asphalt pavement repair, traffic operation and safety driving are mainly considered, and the main maintenance method adopted is to use a new mixture to repair damage on the old asphalt pavement [1]. The repair level of pavement damage is varied due to the restrictions by the actual construction conditions. After the pavement pothole repair, the color difference between the new material at the repaired part and the original pavement is great because the old pavement is exposed for a long time and thus aged. When the influences of certain factors, such as driving environment, are uncertain. The color difference on the pavement has a direct bearing on whether the drivers can accurately judge the pavement damage, and their visual errors impact the behavior decisions. Under high-speed driving state, the color difference after the pothole and crack repair greatly influences the vision and psychology of drivers. Given this situation, the drivers' coping states under the pavement color difference should be reasonably evaluated, and the influencing degree of color difference on drivers' safety driving should be quantified.

In recent years, the number of casualties triggered by traffic accidents has been rising, accompanied by the enormous direct property losses. Accordingly, the road traffic safety status has aroused extensive social attention. Numerous scholars have explored the driving behavior data to better understand the causes for road accidents and proposed that the factors related to road environment account for approximately $28 \%-34 \%$ in urban road traffic accidents [2]. The drivers' decisions and behaviors in traffic environment and their decision-making time to traffic accident are closely related to traffic safety, and $14 \%$ - 34\% of traffic accidents are closely correlated with drivers' attention. According to relevant analysis, the observation errors account for $54 \%$ in traffic accidents; the accidents caused by this factor occupy over a half, and their decisions and behaviors can be considerably affected by their emotions [3]. Therefore, the outstanding color difference element on road motivates drivers' expectations on the road, and the color difference at the pavement repair part influences drivers' perception of road disease status and pavement conditions via the driving expectations to induce road traffic accidents. In the existing criteria for pavement evaluation, the index "road flatness" is mainly considered. However, the vehicle deceleration or evasion caused by the color difference left after the pavement repair harms drivers' physiological and psychological states, thus going against the safe driving and degrading the road traffic capacity.

With the research and development (R\&D) and application of physiological and psychological test instruments, the importance has been attached to drivers' visual and psychological studies in the traffic field. The new-type test instruments have provided effective quantitative and analytical means for scholars to deeply explore the relationship between human state and pavement environment. Many scholars have been seeking for the effective factors that influence drivers in the driving process and the driving environment to correctly understand drivers' attention distribution behavior while driving and acquire effective information of attention distribution. These scholars also paid attention to outstanding elements of road environmental changes. Accordingly, the pavement landscapes and traffic signs have been analyzed, and a certain research progress has been achieved. However, the visual color difference that exists after the pavement damage repair reduces the overall road coordination and contradicts with the original intention beautiful appearance in the pavement design, which not only affects the city image but also induces drivers to visually and psychologically generate scenario 
misjudgement. Drivers need to take a long time in processing information and miss the right moment due to the environmental factors, thus triggering traffic accidents. With the improvement of highway traffic service level and transportation safety operation level, the requirement for road includes not only the traffic function but also the traffic safety. The road readability depends on the complexity of road pavement environment, which is a key factor used to judge the driving safety. The clarity of road information and comfort of pavement users should be fully considered in the maintenance and repair work of pavement damage, and the improvement of driving behavior is the key to the driving safety. If a road repair scheme can provide consideration to the repair-induced color difference, then elevating the threshold value of information deviation can reduce the breadth of its occurrence and contribute to the enhancement of driving stability and safety.

On this basis, the drivers' visual characteristics and psychological changes under the color difference after the pavement repair were demonstrated through a large quantity of real vehicle test data. New requirements and evaluation indexes were proposed for the maintenance work to lay a foundation for further improving the traffic safety.

\section{STATE OF THE ART}

The asphalt pavement damage problem is increasingly prominent, and pothole, which is one of the common and hardest-to-repair diseases of asphalt pavement, has been investigated by many scholars worldwide from various aspects, such as repair structural theory, repair material type, and adhesive property. Some repair schemes have been proposed. However, these studies were mainly concerned with the evaluation indexes of pavement performance. The pavement evaluation methods were based on simple indexes related to surface characteristics to repair the basic traffic function of road [4]. Gao et al. [5, 6] established a 3D finite element model, proposed reasonable combination forms of pothole repair materials and structures, and provided a theoretical basis for selecting the repair materials and structures for potholes with different damage degrees. Scholars have explored the recognition, classification, and impact assessment of pavement damage because it generates adverse effects on vehicle running. Sha et al. [7, 8] introduced the convolutional neural network technology into the image analysis-based pavement disease recognition and measurement and used 2D images to improve the calculation precision and efficiency of the pavement disease. The asphalt pavement disease acquisition technology, which has been digitalized, mainly conducts the feature extraction of pavement cracks and pothole images. However, the extraction and recognition work of color features of diseases, such as potholes, are yet to be carried out. In the above-mentioned studies, the pavement performance, adhesion effect, applicable environment, and repair technology of asphalt disease repair materials are taken as the key research objectives, the service life of pavement is lengthened, and the traffic function is satisfied. Nevertheless, the color difference between the repair material and the original pavement material should be considered to ensure that the driving vision can realize the driving comfort and safety after the repair. The visual discrepancy caused by patches, which are outstanding elements in road, can easily be mixed with the pavement damage, which may result in scenario misjudgment and affect the drivers' traffic safety.

Numerous scholars $[9,10]$ used a driving simulator to study the highway landscapes, traffic signs, and pavement color for deeply exploring the influences of road elements on safe driving. Jiang et al. [11] acquired the driving performance of 33 drivers on six highways via driving simulation. The results showed that the landscapes with 3D branches and leaves were better for the drivability of lowgreen landscape conditions, and moderate greenness and complexity were the best for the drivability. Zhao et al. [12, 13] explored the safety of raised pavement markers (RPM) in expressway tunnel via a driving simulator. They indicated that the RPM could mitigate the drivers' anxiety in dark and monotonic tunnel driving environment and improve their alertness and speed consciousness. However, the driving risk was increased with the increase in tunnel length. Mario-Flores et al. [14] obtained data of stresses and strains through the static, bending, and driving tests by evaluating the influence of cracks on the bus floor on the vehicle safety. They proved that edges and potholes were the key factors that lead to the maximum stress level. Lu et al. $[15,16]$ collected human eye movement data through the driving simulation test. The test data showed that the subtasks lengthened the drivers' takeover response time, no matter under normal driving or cognitive distracted driving. Moreover, the saccade behaviors were always within medium and small ranges. In comparison with normal driving, the drivers' stress response time was lengthened under the cognitive distracted driving, which narrowed the visual search range of drivers.

The above-mentioned studies have stayed in the simulation test phase, while the drivers' conditions under natural driving state have not been studied, not to mention to study the influencing factors of distracted driving. To date, some scholars have conducted real vehicle tests. Bassani et al. [17-20] proposed that the attention mainly depended upon the road environment by studying the visual reaction of drivers under different weather conditions, and speed was an important parameter when the road environment was changed. The road environment generated significant effects on driving speed and attention span and pointed out that the occurrence probability of road traffic accident was high at dusk. Ryu et al. [21, 22] explored the drivers' differences in the gaze region, gaze target, visual load, and visual fatigue under in-vehicle and out-of-vehicle environments. They stated that the information from the central vision was more important than that from the surrounding vision, thereby indicating that the human visual sense organ could be easily affected by external factors. Zhang et al. [23] studied drivers' car following behaviors on highways in foggy environment and conducted the real vehicle test in the real foggy environment. The results showed that the drivers' saccade speed greatly fluctuated in the foggy environment. With a high average saccade speed, the transfer rate of the drivers' points of attention was lower than that in fine weather, and the visibility had a remarkable influence on the visual features of drivers following cars. 
Drivers could be affected by the complexity of road environment and the amount of information. Mao et al. [24, 25 ] analyzed the regular frequency for drivers to encounter damaged road pavement and their unhealthy emotions arising out of this through the real vehicle test. They pointed out that a driver would experience obvious heart rate fluctuation when a pavement damage was suddenly found. Finally, they qualitatively analyzed the influences of pavement damage on vehicle running safety and comfort. The relationship of drivers' psychological and physiological reaction with their driving behaviors has been increasingly explored in the traffic field with the technological development. The present studies regarding the pavement color have mainly concentrated on the influences of different pavement colors on human visual fatigue. However, no experimental measurements and results are available with respect to the influences of the color difference trace after the report on drivers' visual characteristics, physiological indexes, and environmentvision-driving behavior coupling relations. Therefore, the relationship between pothole color difference and drivers' characteristics must be studied. The research on the influences of color difference at the repaired part on drivers' visual characteristics during the real vehicle driving process remains to be urgently carried out.

The driving safety and comfort are no negligible. The asphalt pavement after the pothole repair was taken as the study object to address the deficiencies of the existing studies. A large number of experimental data were acquired using the eye tracker and physiograph, based on the visual cognition behaviors of drivers at different ages when passing through the pothole repair part at different running speeds on different road sections. This task was conducted to figure out the influencing degree of the pothole color difference on drivers' safe driving and threshold value of heart rate and lay a foundation for further enhancing the traffic safety.

The remainder of this study is organized as follows. Section 3 introduces the methods and conditions of test data acquisition. Section 4 analyzes and discusses the results of the visual and psychological tests. Section 5 summarizes the study and draws related conclusions.

\section{METHODOLOGY}

\subsection{Basic Experimental Survey}

\subsubsection{General Survey on the Visual Effect of Pavement Color Difference}

The basic survey before the experiment was completed in the form of questionnaire survey. Besides the influencing degrees of color difference after the pavement disease repair on drivers' vision and psychology, the universality of respondents should also be considered. Hence, this questionnaire was mainly divided into two parts: basic information of drivers, including drivers' gender, age, educational level, and driving years; psychological influences of color difference after the pavement repair on drivers under different driving environments. In the survey, data collection was conducted via network questionnaire, and the respondents were different in terms of region, age, educational level, and driving years. During the survey period, 742 valid questionnaires were collected, and the data were organized and typed in SPSS software, followed by a statistical analysis of drivers' psychological effects generated by the color difference with respect to the traffic safety.

\subsubsection{Visual and Psychological Effect Analysis of Color Difference}

(1) The basic information is illustrated in Tab. 1. Among the drivers accepting the survey, the novice and experienced drivers followed a balanced distribution, with 384 males and 358 females. From multiple aspects, the drivers were widely distributed in terms of age and educational level, each class accounted for a certain proportion, and the distribution of number of drivers was uniform in each class.

Table 1 Basic information of drivers in the survey

\begin{tabular}{|c|c|c|c|}
\hline \multicolumn{2}{|c|}{ Basic information } & $\begin{array}{c}\text { Number of } \\
\text { drivers }\end{array}$ & Percentage / \% \\
\hline Gender & Male & 384 & 51.8 \\
\hline \multirow{2}{*}{ Age } & $20-40$ & 277 & 72.1 \\
\hline & $40-60$ & 107 & 27.9 \\
\hline $\begin{array}{c}\text { Educational } \\
\text { level }\end{array}$ & $\begin{array}{l}\text { Bachelor or } \\
\text { above degree }\end{array}$ & 322 & 83.8 \\
\hline Driving years & Below 3 years & 141 & 36.7 \\
\hline \multicolumn{2}{|c|}{ Professional driver } & 120 & 31.3 \\
\hline Car model & Sedan car & 174 & 45.3 \\
\hline Gender & Female & 358 & 48.2 \\
\hline \multirow{2}{*}{ Age } & $20-40$ & 236 & 69.5 \\
\hline & $40-60$ & 122 & 34.1 \\
\hline $\begin{array}{c}\text { Educational } \\
\text { level }\end{array}$ & $\begin{array}{c}\text { Bachelor or } \\
\text { above degree }\end{array}$ & 269 & 75.1 \\
\hline Driving years & Below 3 years & 197 & 55 \\
\hline \multicolumn{2}{|c|}{ Professional driver } & 34 & 9.49 \\
\hline Car model & Sedan car & 255 & 71.2 \\
\hline
\end{tabular}

(2) The chi-square values $\left(\chi^{2}\right)$ of $3.24,5.12,7.63,11.29$, $6.88,4.23,8.21,10.26,5.23,6.19,10.25$, and 13.68 were obtained through the statistical test of correlations of drivers' gender, age, and educational level with the psychological effects of four road sections in Tab. 2 .

Table 2 Data statistics of psychological influences of pavement color on drivers

\begin{tabular}{|c|c|c|c|c|}
\hline \multirow{2}{*}{$\begin{array}{l}\text { Road section } \\
\text { type with } \\
\text { pothole repair }\end{array}$} & \multicolumn{4}{|c|}{ Influence degree of human psychological effect } \\
\hline & $\begin{array}{c}\text { Serious } \\
\text { influence }\end{array}$ & $\begin{array}{l}\text { Relative } \\
\text { influence }\end{array}$ & $\begin{array}{l}\text { Minor } \\
\text { influence }\end{array}$ & $\begin{array}{c}\text { No } \\
\text { influence }\end{array}$ \\
\hline Expressway & 387 & 252 & 85 & 18 \\
\hline $\begin{array}{c}\text { Tunnel entrance } \\
\text { and exit }\end{array}$ & 346 & 312 & 76 & 8 \\
\hline $\begin{array}{c}\text { Steep road } \\
\text { section }\end{array}$ & 361 & 272 & 96 & 13 \\
\hline Urban road & 165 & 223 & 313 & 41 \\
\hline
\end{tabular}

The test indicates that the drivers' color psychological effects are not significantly different in three factorsgender, age, and educational level. The analytical results show that drivers' visual and psychological effects are universal.

According to the questionnaire data statistics of the visual and psychological effect analysis of color difference, the color difference had a minor effect on drivers' psychology when driving on urban roads. However, over $86.7 \%$ of drivers think that they are relatively influenced when driving at high speed or on a steep road section or at a tunnel entrance. Hence, the above-mentioned three types of road environments were mostly selected for the test road sections in this study. Approximately $78.5 \%$ of occupational drivers deemed that the color difference 
generated by the pavement pothole repair would lead to the misreading of road information. Moreover, the visual information error has a great impact on the concentration of visual attention and brought potential hazards to safe driving.

\subsection{Acquisition of Test Data}

The asphalt pavement pothole repair environment was mainly selected in this test. Different drivers were chosen to participate in the real vehicle test. The visual parameters and physiological indexes of drivers when driving on different road sections with potholes were directly acquired. The equipment was set in advance to maximize the efficiency.

\subsubsection{Testing Equipment}

(1) TobiGlasses 3 eye tracker. This device is a wearable four-sensor eye tracker produced by Jinfa Scientific Research Institution, with an acquisition frequency of 100 $\mathrm{Hz}$, and a full high-definition scenario camera with $106^{\circ}$ combinational field of view.

(2) ErgoLAB intelligent wearable physiograph. This device is a multi-parameter comprehensive detector wearable at various parts of human body and developed and produced by Jinfa Scientific Research Institution. In addition, this tool can implement real-time detection of the physiological indexes, such as respiratory rate, heart rate, electrocardiographic (ECG) change, and electrodermal activity change.

(3) Test vehicle: one Ford Focus car.

(4) Other materials and tools: vehicle data recorder, global positioning system, camera, computer, measuring tape, and second chronograph.

\subsubsection{Test Objects}

According to the test requirements, the main study objects were drivers, and one eye movement test operator and 30 drivers were totally selected through the data processing and screening. The data collected from 20 drivers were finally chosen in the test for analysis, and the influences of gender, age, educational level, character, and driving habit on drivers were not considered. The drivers were divided into two types according to driving years: type A drivers who were novice drivers with less than 3 driving years and type $\mathrm{B}$ drivers who were experienced in driving with over 3 driving years. The drivers of each type drove twice along the surveyed route, and the mean values of test data were taken.

\subsubsection{Determination of the Test Time and Test Road Section}

The real vehicle test was mainly conducted during 09:00 - 17:00 every day under different weather conditions according to the repair conditions of real road. The driving route was planned before the test, and the pavement disease repair conditions were calculated and recognized, including the pothole position, data statistics, and calibration of new and old color difference grades after the repair.

Lyuliang Mountain highway was selected in this test, and some repaired road sections of G209 and S340 were chosen as the test road sections for driving under natural environment. The influences of road elements on the drivers' eye movement characteristics were mainly considered in the test. Hence, different road sections with a large quantity of potholes and cracks repaired were selected, including specific landforms, such as rugged road and tunnel entrance.

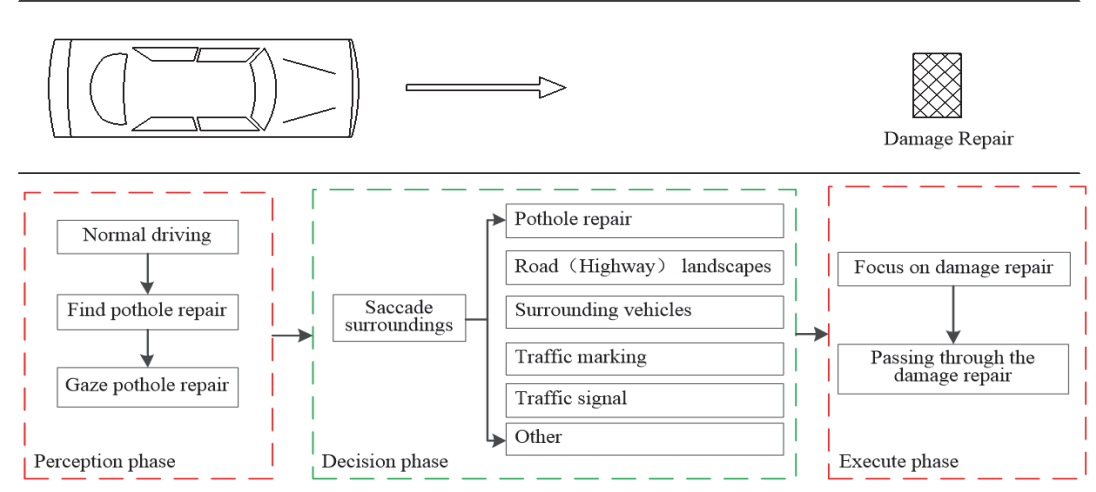

Figure 1 Visual cognition process of drivers for pavement damage repair

\subsubsection{Testing Process}

The drivers participated in the real vehicle test in a real traffic environment wearing the eye tracker and physiograph. Specifically, the drivers freely drove within a specified speed according to the pre-designed route, while their driving habits and driving speed were not restricted. The test data were acquired in the whole process, including their eye movement data not only in normal driving but also in normal parking process, such as congestion, avoidance, and red light. The driving adaptive training was first conducted for 30 minutes before the test (i.e., "double- blind" test) to avoid the influences of the abnormal environmental factors on drivers and data acquisition system. The real intention of the test and changes in the test conditions were not informed to the drivers in advance. The drivers were required to take a good rest the day before the test, and they were forbidden to take any drug or drink any stimulating beverage within $24 \mathrm{~h}$ or do any aggravating activity $2 \mathrm{~h}$ before the test.

When finding a pavement repair trace, the drivers would ignore the repair if they could accurately judge that the repair had no influence or was not in the lane. If the judgment could not be accurately made, then they would 
pay continuous attention to the repair morphology and repair position and change the wheel path. The repairinduced color difference could more easily generate visual errors and doubts, resulting in high importance degree attached by the drivers and longer gaze time. According to the observation results, the drivers would further collect the pavement information through visual, auditory, and tactile senses after judging that the color difference might influence the vehicle running. When driving close to the repair section, the drivers would maintain their attention to the real repair information all the time until completely judging and passing through the damage repair part (Fig. 1).

\section{TEST RESULT AND ANALYSIS \\ 4.1 Visual Cognition of Color Difference after the Pavement Pothole Repair}

Under intricate traffic environment, drivers may be stimulated by all types of traffic information, and they must accurately perceive the traffic information and rapidly make a correct choice, namely, identify the important targets, take reasonable measures, and ensure the safe vehicle running. During the driving process, the drivers acquire the traffic information mainly through the visual sense, accounting for over $80 \%$ of all information. Therefore, the drivers' recognition characteristics in the driving process are the basic information used to study driving behaviors.

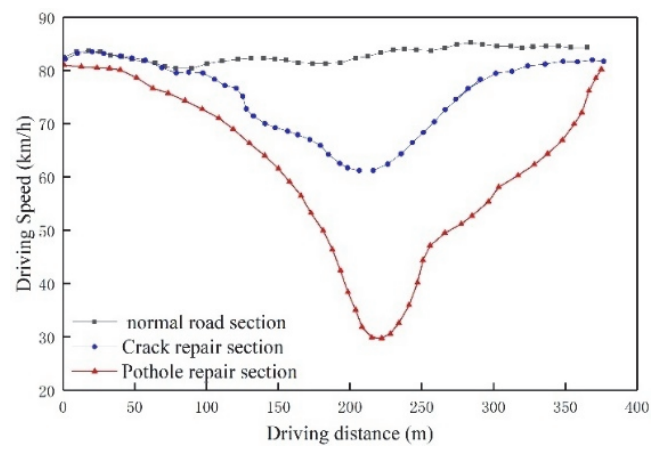

Figure 2 Changes in vehicle driving speed under different road section conditions

Twenty groups of effect test data were collected, and the length of the pothole repair section was recorded. The normal road section and cracking section with the same length were intercepted to compare drivers' differences in driving speed. Fig. 2 shows that the speed of the vehicles on the normal and cracked sections slightly changes in the process of test. Meanwhile, the speed of the vehicles on the road section with potholes is greatly changed, showing an obvious declining trend on the pothole pavement. This phenomenon indicates that the potholes have an obvious influence on drivers' driving speed.

\subsubsection{Distribution Characteristics of Points of Gaze}

The number of gaze times is an important index reflecting drivers' information search and processing efficiency and the importance degree of region of gaze. The greater the number of gaze times, the more important the region of driver gaze. Fig. 3 shows that the drivers' field of view is divided into seven regions. Region 1 represents the left far sight. Region 2 denotes the far sight right ahead. Region 3 is the right far sight. Region 4 is the left near sight and left rear sight. Region 5 is the near sight right ahead. Region 6 is the right rear sight. Region 7 is the region of in-car driver's gaze.

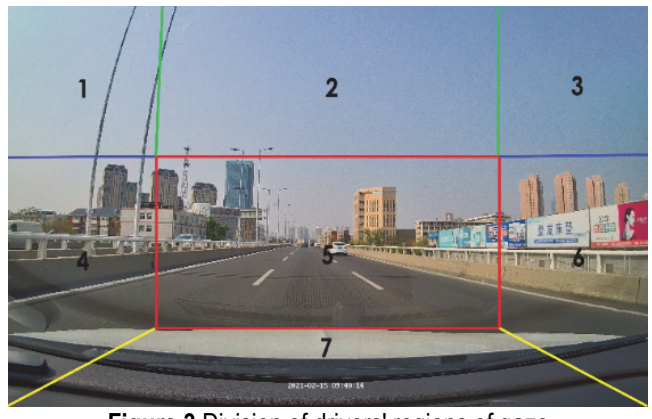

Figure 3 Division of drivers' regions of gaze

According to the survey and statistics, the main region of drivers' gaze is Region 5, followed by Region 2 (Tab. 3). Comparison with the normal road section, the points of drivers' gaze on the road section experiencing the pavement repair are more concentrated in the near sight regions (Regions 4, 5, and 6), which is especially obvious on the asphalt pavement section experiencing the pothole repair. The gaze frequency in Region 4 is increased by $11.5 \%$ when the vehicle is running on the road section with potholes compared with the normal road section. In the visual search, the number of regional points of gaze and gaze time are used to measure the search efficiency, thereby indicating a certain complexity after the pavement pothole repair. Moreover, the drivers enhance their attention to the pavement damage repair part.

Table 3 Drivers' gaze frequency in each region (Unit: \%)

\begin{tabular}{|c|c|c|c|}
\hline $\begin{array}{c}\text { Region of } \\
\text { gaze }\end{array}$ & $\begin{array}{c}\text { Normal road } \\
\text { section }\end{array}$ & $\begin{array}{c}\text { Crack repair } \\
\text { section }\end{array}$ & $\begin{array}{c}\text { Pothole repair } \\
\text { section }\end{array}$ \\
\hline 1 & 3.29 & 2.78 & 2.18 \\
\hline 2 & 9.76 & 6.18 & 4.29 \\
\hline 3 & 5.15 & 4.31 & 3.25 \\
\hline 4 & 5.01 & 5.19 & 5.27 \\
\hline 5 & 70.22 & 74.87 & 78.35 \\
\hline 6 & 3.88 & 4.21 & 4.62 \\
\hline 7 & 2.78 & 2.46 & 2.04 \\
\hline
\end{tabular}

The coordinate scatter diagram of points of gaze of normal road section and pothole road section are shown in Fig. 4 and Fig. 5, the circles represent the points of gaze, and the area of circle denotes the accumulative gaze time. Fig. 4 and Fig. 5 demonstrate that the number of points of drivers' gaze is relatively small on the normal road section.

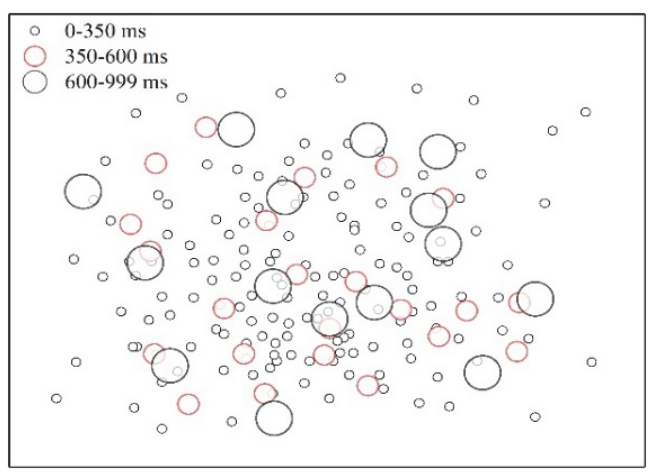

Figure 4 Coordinate scatter diagram of points of gaze normal road section 


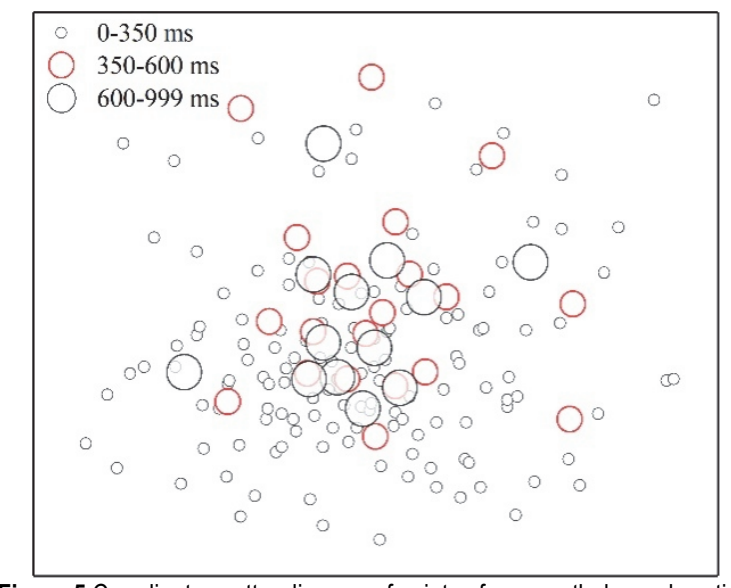

Figure 5 Coordinate scatter diagram of points of gaze pothole road section

The quantity of points of gaze on the pothole repair section is greater than that on the normal road section, and drivers are mainly concentrated within one region. Meanwhile, the quantity of points of gaze in the concentrated region and the accumulative gaze time are increased. The number of gaze times reflects the difficulty level for the drivers to read the pavement information, and the gaze time represents the correct information reading efficiency. When driving on the pothole repair section, the drivers increased their number of gaze times at the repaired part and lengthened the gaze time at the pavement potholes, thereby manifesting the great importance attached to the pothole regions. The color difference information after the pothole repair would result in a great scenario misjudgment among the drivers. This notion means that the more complex the regional information, the more doubtful the drivers are over this point, and the greater the influence degree on the driving safety.

\subsubsection{Saccade Characteristics}

Saccade range includes angle between the lines of sight corresponding to any two adjacent points of gaze. The drivers' saccade ranges on two different pavements are shown in Fig. 6 through the data arrangement. Saccade range reflects the tension in the visual information acquisition, and the number of saccade times reflects the drivers' familiarity with the external environment. The survey indicates that the number of small-range saccade behaviors of drivers is greater than that of large-range saccade behaviors no matter whether the pavement environment is complex or not. The drivers' saccade range on the normal road section is mainly concentrated within $10^{\circ}-50^{\circ}$, with the average saccade range of $24.76^{\circ}$. The saccade range on the damaged road section is mainly concentrated within $0^{\circ}-28^{\circ}$, with the average range of $15.72^{\circ}$; thus, obvious differences are manifested. This situation occurred because the drivers could generate the scenario misjudgment due to the vision difference caused by the color difference at the pothole repair part. Drivers would pay more attention to the pavement potholes in front until safely passing through the section through the accurate judgment. During the driving process, the saccade range and the drivers' attention to the surrounding environment were reduced.

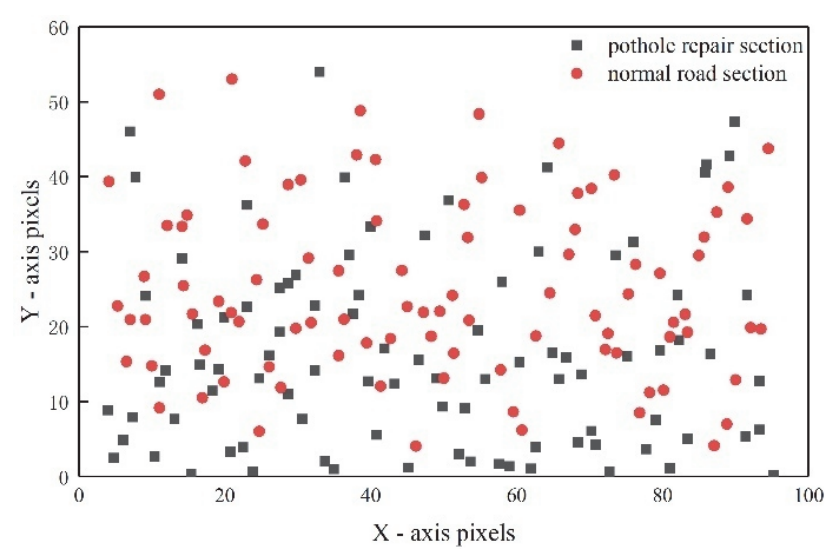

Figure 6 Drivers' saccade range

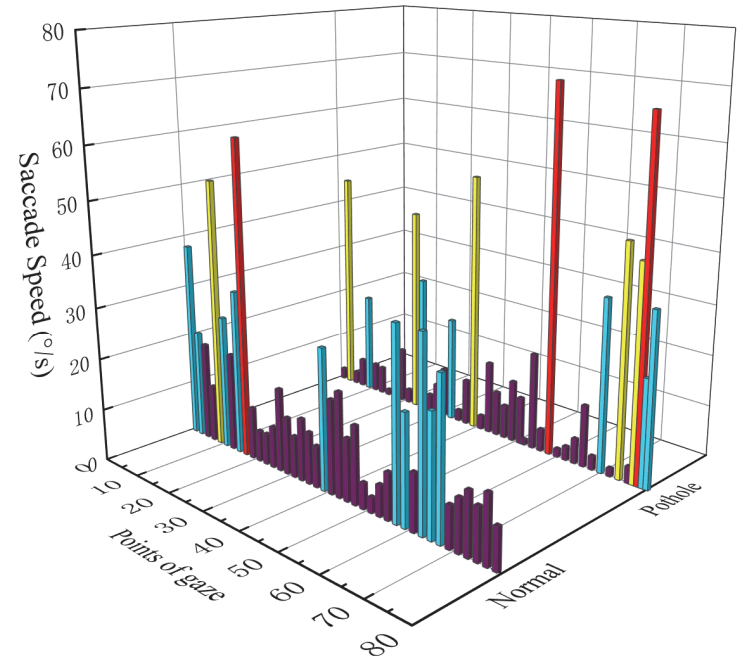

Figure 7 Distribution of the number of drivers' saccade times-saccade speed when on two pavement types

Saccade speed is the average transition speed between points recorded by the eye tracker (Fig. 7). Under the normal pavement environment, the saccade ranges smaller than $10^{\circ}$ account for approximately $73.5 \%$. The drivers' saccade speed approximately follows the logarithmic normal distribution. The speeds of over $87 \%$ of saccade behaviors range from $0 \%-100 \%$ s. When the pothole repair trace exists on the pavement, the low-speed saccade behaviors are reduced, while the high-speed ones are increased. The saccade speed reflects the quantity of visual information acquired and the information capturing and processing efficiency. This notion indicates that the higher the saccade speed, the tenser the drivers would feel during the driving process. Moreover, the drivers' feeling of tension is enhanced when passing through the pothole pavement.

\subsubsection{Pupil Characteristic Analysis}

The changes in drivers' pupil during the driving process are seen in Fig. 8. The change records $25 \mathrm{~s}$ before and after passing through potholes are chosen. Within the 10 - $15 \mathrm{~s}$, the drivers' pupil areas greatly changed on the pothole section, and the change amplitude was reduced when they passed through the normal pavement, which was similar to the change in the pupil area when the drivers passed through the pavement with crack repair trace. 


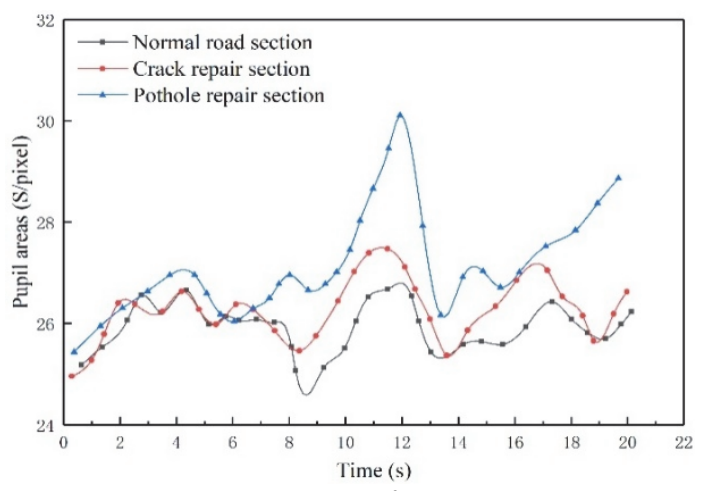

Figure 8 Time-dependent changes of drivers' average pupil areas

Under different road element environments, the average pupil diameter shows various changes with the increase in the information quantity and complexity. The pupil diameter is enlarged when the light intensity is basically identical, thus indicating that the human body felt the increasing psychological and physiological pressures generated by the external environment. Although the average pupil diameter is enlarged on the road section with pothole repair trace, the discreteness is relatively small, thereby manifesting that drivers attempted to fix at the target (pothole repair part) under this environment. When the elements of this type discontinuously appeared on the road section, the psychological tension caused by the information of the external visual stimulus reflected the conditioned response generated by the human body to the external environmental changes. During the driving process, the driving process is safe if the change rate of the drivers' pupil area is within $20 \%$, and they are under a comfortable visual state. When driving on the pothole pavement, the frequency at which the drivers' pupil area fluctuates by over $20 \%$ reaches $1 / 5$, and the drivers' visual load is strengthened, thus easily generating the feeling of fatigue.

\subsection{Drivers' Physiological Indexes on the Repaired Road Section}

The heart rates of types A and B on the normal and test road sections are tested as seen in Fig. 9 (the heart rate in the figure is the mean value per minute). The black and blue curves represent the change curves of the heart rates of type A and B drivers on the normal road section, respectively. The red and green curves denote the change curves of their heart rates on the pothole road section, respectively.

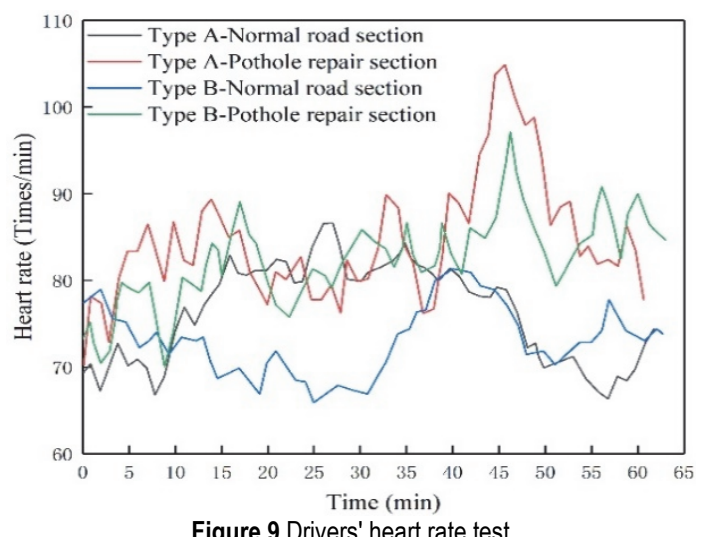

From the drivers' heart rate test in Fig. 9, the mean and maximum values of the drivers' heart rate on the normal road section are 74.65 and $86.61 \mathrm{times} / \mathrm{min}$, and those on the road section with potholes are 83.13 and 104.87 times/min, which are increased by $11.3 \%$ and $21.1 \%$, respectively. This notion indicates that the psychological tension in the driving process on the pothole repaired road section is higher than that on the normal road section. From the angle of driver type, the overall spread values of the heart rate curve of type $\mathrm{B}$ drivers on the normal road section and pothole section are 74.30 and 82.41 times $/ \mathrm{min}$, respectively. Under the same environment, the overall spread values of type A drivers are greater than those of type B drivers by $2.83 \%$ and $5.81 \%$, respectively. The novice and experienced drivers are not different in the heart rate on the normal road section. However, the heart rate of all drivers would experience great fluctuations under the driving state on the pothole section. The mean overall spread of novice drivers' heart rate curve is greater than that of experienced drivers, thereby manifesting that the heart rate fluctuation of novice drivers is greater, and they could easily feel tense.

The ECG signal belongs to a random signal, the heart rate difference will still exist among the drivers under the same circumstance, and the individual physical levels also vary. The heart rate variation cannot comprehensively reflect the influencing degree of road environment on the drivers' psychology and physiology. Hence, the heart rate growth rate (ratio of difference value between a driver's heart rate on pothole section and that on normal road section to the heart rate on the normal section) is proposed. The heart rate changes of two types of drivers when driving through the pothole repaired section at different speeds are continuously measured using an electrocardiograph to analyze the correlations of vehicle speed and heart rate with the pavement information. The heartbeat data saved by the electrocardiograph are transformed into the heart rate data. The driving records, such as overtaking and meeting, are excluded by maintaining the uniform contrast of recording time to vehicle speed. The vehicle speed and monitored heart rate within 1 minute before and after each pothole are taken. The offset data should be eliminated to mitigate the influence of adverse noise. The mean values of vehicle speed and heart rate growth rate of different drivers in various time periods are displayed in Tab. 4.

The regression analysis of the sample data in Tab. 4 is conducted. Then, the variation tendency chart (Fig. 10) of the vehicle speed and heart rate growth rate on the pothole repaired road section is obtained. The analysis indicates that the vehicle speed per hour is correlated with the drivers' heart rate on the pothole repaired road section. When the vehicle speed is approximately $40-70 \mathrm{kmh}^{-1}$, the correlation between the heart rate growth rate and the vehicle speed is low. The variation trend of heart rate growth rate is not apparent with the acceleration of the vehicle speed, being largely under a discrete distribution within the range of $7 \%-18 \%$. When the vehicle speed is approximately $70-110 \mathrm{kmh}^{-1}$, the heart rate growth rate basically presents a linear growth relation with the vehicle speed, namely, the heart rate growth rate is on the sharp increase with the vehicle speed. 


\begin{tabular}{|c|c|c|c|c|c|c|c|c|}
\hline \multirow{2}{*}{$\begin{array}{r}\text { Driver type } \\
\text { Vehicle speed } V /\left(\mathrm{km} \cdot \mathrm{h}^{-1}\right)\end{array}$} & \multicolumn{4}{|c|}{ Type A driver } & \multicolumn{4}{|c|}{ Type B driver } \\
\hline & 43.67 & 57.36 & 50.95 & 41.53 & 40.52 & 45.32 & 56.58 & 53.19 \\
\hline Heart rate growth rate $(\%)$ & 9.36 & 13.26 & 14.28 & 8.75 & 8.33 & 13.59 & 15.83 & 9.62 \\
\hline Vehicle speed $V /\left(\mathrm{km} \cdot \mathrm{h}^{-1}\right)$ & 62.13 & 76.95 & 63.67 & 61.48 & 63.02 & 75.13 & 64.78 & 79.87 \\
\hline Heart rate growth rate $(\%)$ & 10.21 & 23.84 & 14.27 & 11.64 & 9.56 & 10.25 & 18.93 & 26.73 \\
\hline Vehicle speed $V /\left(\mathrm{km} \cdot \mathrm{h}^{-1}\right)$ & 83.45 & 93.47 & 95.78 & 83.27 & 92.13 & 98.23 & 83.59 & 89.05 \\
\hline Heart rate growth rate $(\%)$ & 22.19 & 33.56 & 38.83 & 28.56 & 30.22 & 30.65 & 24.59 & 25.41 \\
\hline Vehicle speed $V /\left(\mathrm{km} \cdot \mathrm{h}^{-1}\right)$ & 104.36 & 112.98 & 102.13 & 108.41 & 111.15 & 101.16 & 113.13 & 109.63 \\
\hline Heart rate growth rate $(\%)$ & 36.23 & 46.29 & 40.46 & 48.72 & 38.26 & 39.16 & 40.21 & 37.86 \\
\hline
\end{tabular}

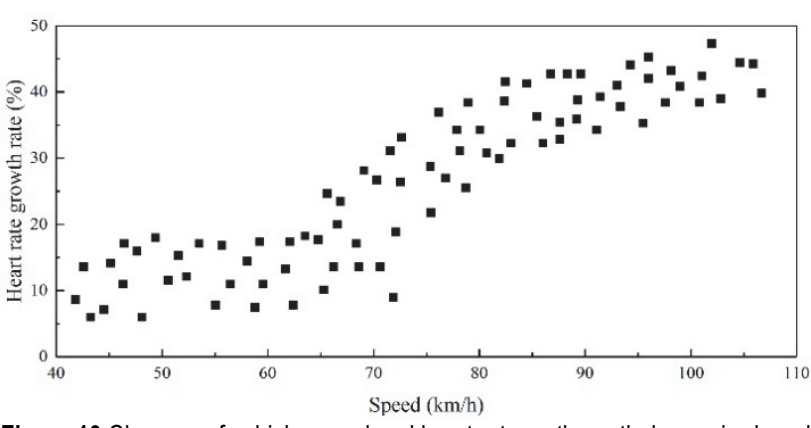

Figure 10 Changes of vehicle speed and heart rate on the pothole repaired road section

The accumulative frequency curves of the heart rate growth rate are seen in Fig. 11 through the grouping statistics of the data in Tab. 4. The drivers' different driving years and their cognitive degrees exert different effects on the heart rate. In the test of type A drivers, the heart rate variation of $89 \%$ of novice drivers exceeds $20 \%$. When the heart rate growth rate is within $25 \%-40 \%$, the growth trend would be changed, the accumulative frequency is increased to $90 \%$, and the growth rate could reach as high as over $50 \%$. In the test of type B drivers, the accumulative frequency is approximately $60 \%$ when the heart rate growth rate is within $23 \%-25 \%$. The growth is rapid, the accumulative frequency already reaches $81 \%$, and the maximum heart rate growth rate is approximately $41 \%$ when the heart rate changes within $25 \%-30 \%$. The heart rate growth rate of type $A$ drivers is apparently greater than that of type B drivers, and the overall spread heart rate is $7.2 \%$. Under general circumstances, most drivers are quiet and prudent in the driving process. This moderate tension is a normal psychological reaction. On the pothole repaired road section, the drivers' psychological tension is changed when the post-repair color difference is an uncertain external factor. The heart rate growth of novice drivers is more obvious. When passing through the pothole section, the heart rate growth rate of $22 \%$ of drivers exceeds $40 \%$. Although this state accounts for a small proportion in the driving process, it could easily lead to accidents.

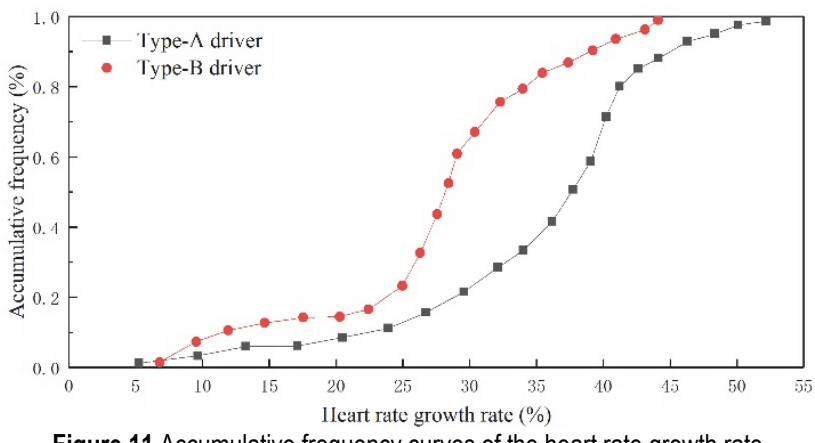

Figure 11 Accumulative frequency curves of the heart rate growth rate
$V_{85}=91 \mathrm{~km} / \mathrm{h}$ was calculated through the interpolation method and the data analysis of the vehicle speed and heart rate growth rate. According to the high-speed driving experience, drivers would be in danger when driving at a speed exceeding the above speed value, and the drivers' heart rate growth rate is approximately $36.8 \%$. Thus, this value can be a recommended value used to evaluate the drivers' psychological tension. Given this situation, the circumstance that the heart rate growth rate accounting for a certain proportion is greater than $36.8 \%$ belonged to a critical value that might endanger the driving safety. Meanwhile, the vehicle speed has a significant influence on the drivers' psychology when the vehicle speed is continuously increased, and the maximum heart rate growth rate is approximately $53 \%$, meaning that the drivers are quite psychologically tense, which is extremely bad for the safe driving.

According to the experimental study results and the prominent positions in the accumulative frequency curves of drivers' heart rate growth rate, the heart rate growth rate of $36 \%$ is determined as the physiological evaluation criterion for the drivers' psychological tension under the free flow condition on the pothole repaired road section. Meanwhile, $43 \%$ is the safety threshold value of the psychological limit that should be avoided in the driving process. In the test, the drivers show largely identical psychological and physiological change trends, and their heart rates are elevated to different degrees. The statistical results indicate that the heart rate growth rate exceeds $32 \%$ among $58.5 \%$ of drivers. The study results have shown that the drivers will feel tense when their heart rate growth rate exceeds $30 \%$; if it exceeds $40 \%$, then they will feel quite tense, and dangers can easily take place, which verifies the test results in this study. Accordingly, the drivers' heart rates fluctuate due to the changes in the road environment and drivers' physiological and psychological conditions. During the process of high-speed vehicle's running, the color difference could arouse high attention from the drivers or cause an emergency braking. A certain time delay exists in the information received by drivers because of the concealment and uncertainty of repair information. The longer the processing time it takes to process, the more dangerous drivers will be, resulting in a greater psychological burden. The more nervous the driver is, the higher the heart rate will be.

\section{CONCLUSIONS}

This study aimed to explore the influencing degrees of color difference between new and old materials after the asphalt pavement pothole repair on the traffic operation and improve the safety of driving. From the engineeringpsychology interdisciplinary angle and by means of a real vehicle test, the influence of pavement repair-induced 
color difference on the driving safety was studied on the basis of the analysis of visual information and psychological indexes. The following conclusions were drawn:

(1) Among the drivers of different ages and driving years including occupational drivers, quantity of drivers' points of gaze and gaze time will be increased on the pothole repaired road section. The increase in amplitude is especially large among the novice drivers (less than 3 years of driving), and their frequency of attention to the front route, surrounding vehicles, and traffic signs is lower than that of experienced drivers.

(2) The saccade range of drivers on the pothole section is mainly concentrated within $0^{\circ}-28^{\circ}$ with the average saccade range of $15.72^{\circ}$ being smaller than that on the normal road section. The average pupil diameter is enlarged, the frequency at which the pupil change rate exceeds the safety threshold $(20 \%)$ reaches $1 / 5$, and the drivers' tension is enhanced on the pothole section, which can easily generate fatigue.

(3) The mean and maximum values of pothole repaired road section are greater than those on the normal road section. Under the pothole repair environment and at the vehicle speed lower than $70 \mathrm{~km} \cdot \mathrm{h}^{-1}$, the heart rate growth rate largely presents a discrete distribution within 7\% $18 \%$. When the vehicle speed is higher than $80 \mathrm{~km} \cdot \mathrm{h}^{-1}$, the heart rate growth rate shows a linear growth relation with the vehicle speed. Novice drivers will experience stronger heart rate fluctuations than experienced drivers, especially when the vehicle speed exceeds $75 \mathrm{~km} \cdot \mathrm{h}^{-1}$. The heart rate of novice drivers is rapidly accelerated due to the color difference after the pavement repair. The excessive heart rate growth can easily affect the driving safety.

(4) According to the experimental analysis and calculation and psychological research results, the heart rate growth rate of $36 \%$ is determined as the physiological evaluation criterion for the drivers' psychological tension on the pothole road section and $43 \%$ as the safety threshold of psychological limit that should be avoided in the driving process. Novice drivers are more susceptible to tension, which may lead to potential safety hazards in the driving process.

In conclusion, various sophisticated instruments will be applied in safe driving with the R\&D and application of physiological and psychological test instruments. According to this study, the color difference trace between new and old materials left over after the pothole repair of asphalt pavement will generate great potential safety hazards in the driving process. This work lays a foundation for the subsequent applied research in safe driving. The study results can support the speed limitation on pothole road section under specific environment, evaluation of pavement repair, and comprehensive safe driving guarantee. The follow-up study will be carried out from the aspects of pothole repair generating different color differences and drivers' gender and line form of road section to form a comprehensive research system for the driving behaviors under the pavement color difference and propose higher requirements and more evaluation indexes for the asphalt pavement maintenance and repair link.

\section{REFERENCES}

[1] Yang, Y. H., Yan, X. T., An, Z. H., \& Yang, Y. (2020). Development of Asphalt Pavement Pit Repair Materials and Analysis of Structural Mechanical Response. Journal of Shenyang Jianzhu University, 36(2), 314-322.

[2] Singh, H. \& Kathuria, A. (2021). Analyzing driver behavior under naturalistic driving conditions: A review-Science Direct. Accident Analysis \& Prevention, 150, 1-21. https://doi.org/10.1016/j.aap.2020.105908

[3] Escadas, M., Jalali, M. S., \& Farhangmehr, M. (2019). Why bad feelings predict good behaviours: The role of positive and negative anticipated emotions on consumer ethical decision making. Business Ethics: A European Review, 28(1), 1-17. https://doi.org/10.1111/beer.12237

[4] Papageorgiou, G. (2019). Appraisal of Road Pavement Evaluation Methods. Journal of Engineering Science and Technology Review, 12(6), 158-166. https://doi.org/10.25103/jestr.126.20

[5] Gao, J. (2019). Three-dimensional Pavement Damage Detection and Characteristic Value Comouting Application System. Master's Thesis of Chang'an University, 20-30.

[6] Chen, L. L., Pan, S. Q., Qian, Z D., \& L, G. (2018). Quantifiable field performance evaluation technique of asphalt pavement pothole patching. Journal of Southeast University(Natural Science Edition), 48(06), 1088-1093.

[7] Sha, A. M., Tong, Z., \& Gao, J. (2018). Recognition and measurement of pavement disasters based on convolutional neural networks. China Journal of Highway and Transport, 31(01), 1-10.

[8] Tran, V. P., Tran, T. S., Lee, H. J., Kim, K. D., \& Nguyen, T. T. (2021). One stage detector (RetinaNet)-based crack detection for asphalt pavements considering pavement distresses and surface objects. Journal of Civil Structural Health Monitoring, 11(3), 1-18. https://doi.org/10.1007/s13349-020-00447-8

[9] Peruzzini, M., Pellicciari, M., \& Grandi, F. (2019). A multimodal virtual reality set-up for human-centered design of industrial workstations. DYNA, 94(2), 1-10. https://doi.org/10.6036/8889

[10] Badejo, J. A., Akinrinmade, A. A., \& Adetiba. E. (2019). Survey of Periocular Recognition Techniques. Journal of Engineering Science and Technology Review, 12(5), 214226. https://doi.org/10.25103/jestr.125.24

[11] Jiang, B., He, J., Chen, J., \& Larsen, L. (2021). Moderate is optimal: A simulated driving experiment reveals freeway landscape matters for driving performance. Urban Forestry \& Urban Greening, 58, 126976. https://doi.org/10.1016/j.ufug.2021.126976

[12] Zhao, X. H., Ju, Y. J., Li, H. J., Zhang, C. F., \& Ma, J. M. (2020). Safety of Raised Pavement Markers in Freeway Tunnels Based on Driving Behavior. Accident Analysis \& Prevention, 145,105708. https://doi.org/10.1016/j.aap.2020.105708

[13]Qin, X. C., Zhang, N., Zhang, W. H., Meitner, M., \& Meitner, M. (2020). How does tunnel interior color environment influence driving behavior? Quantitative analysis and assessment experiment. Tunnelling and Underground Space Technology, 98,103320. https://doi.org/10.1016/j.tust.2020.103320

[14] Acosta-Flores, F., Jimenez-Lopez, E., \& Cuenca-Jimenez, F. (2019). Determination and solution to the structural failures in a urban bus. DYNA, 94(1), 100-105.

[15] Lu, G. Q., Zhao P. Y., Wang, Z. J., \& Lin, Q. F. (2018). Impact of visual secondary task on young drivers take-over time in automated driving. China Journal of Highway and Transport, 31(04), 165-171.

[16] Lyu, A. H., Ma, Y., Li, T. F., \& Zhang, Y. L. (2018). Analysis of stress response characteristics of driver with 
cognitive distraction. China Safety Science Journal, 28(07), $7-12$

[17] Bassani, M., Catani, L., Salussolia, A., \& Yang, C. Y. D. (2019). A driving simulation study to examine the impact of available sight distance on driver behavior along rural highways. Accident Analysis \& Prevention, 131(OCT.), 200212. https://doi.org/10.1016/j.aap.2019.07.003

[18] González-Hernández, B., Usami, D. S., Prasolenko, O., Burko, D., \& Persia, L. (2020). The driver's visual perception research to analyze pedestrian safety at twilight. Transportation Research Procedia, 45, 827-834. https://doi.org/10.1016/j.trpro.2020.02.087

[19] Wurthmann, K. (2020). How group and perceiver characteristics affect collective blame following counterproductive work behavior. Business Ethics A European Review, 2, 15. https://doi.org/10.1111/beer.12251

[20] Vetturi, D., Tiboni, M., Maternini, G., \& Bonera, M. (2020). Use of eye tracking device to evaluate the driver's behaviour and the infrastructures quality in relation to road safety. Transportation Research Procedia, 45, 587-595. https://doi.org/10.1016/j.trpro.2020.03.053

[21] Ryu, D., Cooke, A. M., Bellomo, E., \& Woodman, T. (2020). Watch out for the hazard! Blurring peripheral vision facilitates hazard perception in driving. Accident Analysis \& Prevention, 146, 1-13. https://doi.org/10.1016/j.aap.2020.105755

[22] Wang, L., Yu, S. H., \& Chu, J. J. (2020). Position design of in-car information display based on eye movement analysis. Journal of Zhejiang University, 54(04), 671-677+693.

[23] Zhang, X. G., Gao, J. P., Liao, L., \& Deepak, B. (2021) Study on Express way Driver's Car Following Behavior in Fog Environment. China Journal of Highway and Transport, $1-15$.

[24] Mao, L. C. (2013). Research on Vehicle Running Mode \& Characteristics of Traffic Flow Resulting from Pavement Distress. Master's Thesis of Harbin Institute of Technology, 20-40.

[25] Li, X. S., Meng, F. S., Zheng, X. L., \& Ren, Y. Y. (2018). Psychological Characteristics of Drivers in the Stress Scene Based on Heart Rate Variability. Journal of Shanghai Jiaotong University, 52(02), 163-168.

\section{Contact information:}

Hongjun JING, PhD, Professor

(Corresponding author)

1) School of Architectural and Civil Engineering, Xi'an University of Science and Technology, Shaanxi Province, China,

2) Road Engineering Research Center of Xi'an University of Science and

Technology, Shaanxi Province, China,

No.58 Yanta Road, Xi'an City, Shaanxi Province, China

E-mail: jinghongjun@xust.edu.cn

Qian LIU, MS

1) School of Architectural and Civil Engineering, Xi'an University of Science and Technology, Shaanxi Province, China,

2) Road Engineering Research Center of Xi'an University of Science and

Technology, Shaanxi Province, China,

No.58 Yanta Road, Xi'an City, Shaanxi Province, China

E-mail: 19204053022@stu.xust.edu.cn

\section{Meng GAO, MS}

1) School of Architectural and Civil Engineering, Xi'an University of Science and Technology, Shaanxi Province, China,

2) Road Engineering Research Center of Xi'an University of Science and Technology, Shaanxi Province, China

No.58 Yanta Road, Xi'an City, Shaanxi Province, China

E-mail: 19204053013@stu.xust.edu.cn

\section{Jiahao ZHANG, MS}

1) School of Architectural and Civil Engineering, Xi'an University of Science and Technology, Shaanxi Province, China,

2) Road Engineering Research Center of Xi'an University of Science and Technology, Shaanxi Province, China,

No.58 Yanta Road, Xi'an City, Shaanxi Province, China

E-mail:1504050416@stu.xust.edu.cn

\section{Xiao MU, MS}

1) School of Architectural and Civil Engineering, Xi'an University of Science and Technology, Shaanxi Province, China,

2) Road Engineering Research Center of Xi'an University of Science and Technology, Shaanxi Province, China,

No.58 Yanta Road, Xi'an City, Shaanxi Province, China

E-mail: 20204228111@stu.xust.edu.cn 